\title{
A LIMITING VISCOSITY APPROACH TO RIEMANN SOLUTIONS CONTAINING DELTA-SHOCK WAVES FOR NONSTRICTLY HYPERBOLIC CONSERVATION LAWS
}

\author{
BY \\ JIAXIN HU \\ Young Scientists Laboratory of Mathematical Physics, \\ Wuhan Institute of Mathematical Sciences, Academia Sinica, P. R. China
}

1. Introduction. We are concerned with the Riemann problem for

$$
\begin{aligned}
& u_{t}+\left(u^{2}\right)_{x}=0 \\
& v_{t}+(u v)_{x}=0
\end{aligned} \quad(x, t) \in \mathbf{R} \times \mathbf{R}_{+},
$$

with initial values

$$
(u, v)(x, 0)= \begin{cases}\left(u^{+}, v^{+}\right), & x>0, \\ \left(u^{-}, v^{-}\right), & x<0 .\end{cases}
$$

System (1.1) has the two eigenvalues $\lambda_{1}=u$ and $\lambda_{2}=2 u$ with corresponding right eigenvectors $r_{1}=(0,1), r_{2}=(1, v / u)^{\mathrm{T}}$, and $\nabla \lambda_{1} \cdot r_{1} \equiv 0, \nabla \lambda_{2} \cdot r_{2}=2$. Thus (1.1) is nonstrictly hyperbolic and $\lambda_{1}$ is linearly degenerate; $\lambda_{2}$ is genuinely nonlinear.

We recall that the classical Riemann solution is composed of a shock (contact discontinuity) or rarefaction of the slower family followed by a wave of the second. But in the present situation, one finds that no classical weak solutions exist for certain states $\left(u^{ \pm}, v^{ \pm}\right)$, and distributions of the form of Dirac delta shock waves supported on discontinuity lines are found necessary, even though for systems of conservation laws satisfying the classical assumptions (strict hyperbolicity and genuine nonlinearity), the Riemann problem breaks down for some large data [5]. For the two-dimensional system of conservation laws we refer the reader to [10].

System (1.1) with trivial difference $(t \rightarrow 2 t)$ was studied by Korchinski [6] in his Ph.D. Thesis in 1977. Generalized delta-functions were used in his numerical study and in the construction of his unique solution to the Riemann problem. Afterwards, Tan, Zhang, and Zheng [11] introduced a viscosity term in the first equation of (1.1),

$$
\begin{aligned}
& u_{t}+\left(u^{2}\right)_{x}=\varepsilon t u_{x x}, \\
& u_{t}+(u v)_{x}=0
\end{aligned}
$$

and proved that the self-similar solutions $\left(u^{\varepsilon}, v^{\varepsilon}\right)$ to $(1.3),(1.2)$ converge weakly star to delta-shock waves for some states $\left(u^{ \pm}, v^{ \pm}\right)$. In this paper, we employ the limiting

Received September 15, 1994.

1991 Mathematics Subject Classification. Primary 35L65. 
viscosity approach, first introduced by Tupciev [9] and by Dafermos [1], to solve the Riemann problem (1.1), (1.2). As to the applications of this approach, we refer the reader to [2], [7], [8], and [3], etc.

The program in this paper is as follows: in Sec. 2 we show that the viscosity regularized problem

$$
\begin{aligned}
& u_{t}+\left(u^{2}\right)_{x}=\varepsilon t u_{x x} \\
& v_{t}+(u v)_{x}=\varepsilon t v_{x x}
\end{aligned} \quad(x, t) \in \mathbf{R} \times \mathbf{R}_{+}
$$

with initial data (1.2) has a smooth self-similar solution of the form $(u(x / t), v(x / t))$, functions of the single variable $\xi=x / t$. Equivalently, we consider the boundary-value problem

$$
\begin{aligned}
& \varepsilon \ddot{u}=-\xi \dot{u}+2 u \dot{u} \\
& \varepsilon \ddot{v}=-\xi \dot{v}+(\dot{u} v+u \dot{v})
\end{aligned} \quad \xi \in \mathbf{R},
$$

with boundary conditions

$$
(u(-\infty), v(-\infty))=\left(u^{-}, v^{-}\right), \quad(u(+\infty), v(+\infty))=\left(u^{+}, v^{+}\right)
$$

It is shown that $(1.5),(1.6)$ has a smooth solution $\left(u_{\varepsilon}(\xi), v_{\varepsilon}(\xi)\right)$ on $(-\infty, \infty)$ by applying the Leray-Schauder fixed point theorem (see [4, pp. 280-281]). In Sections 3 and 4 we prove that limit solutions of (1.5), (1.6) generate solutions of the Riemann problem $(1.1),(1.2)$. In the resulting solution, $u(x, t)$ is always a bounded monotone function of $\xi=x / t$ while $v(x, t)$ may be unbounded along a single ray $x / t=\tau_{0}$. In particular, when $u^{+} \leq 0 \leq u^{-}, v(x, t)$ consists of a discontinuity line $x / t=u^{+}+u^{-}$plus a distributional weighted Dirac delta function with $x / t=u^{+}+u^{-}$as its support. At the same time, $u(x, t)$ is required to take certain values on $x / t=u^{+}+u^{-}$, so that (1.1) holds in the sense of distributions.

One can verify that $(u(x / t), v(x / t))=(u(\xi), v(\xi))$ is a solution to (1.1), (1.2) if and only if

$$
\begin{aligned}
-\xi \dot{u}+2 u \dot{u} & =0, \\
-\xi \dot{v}+(\dot{u} v+u \dot{v}) & =0
\end{aligned}
$$

holds in the sense of distributions and $(u(\xi), v(\xi))$ satisfies $(1.6)$. Thus, if $\xi_{0} \in(-\infty, \infty)$ is a discontinuity point of $(u(\xi), v(\xi))$, the Rankine-Hugoniot conditions become

$$
\begin{aligned}
\xi_{0} & =u\left(\xi_{0}+0\right)+u\left(\xi_{0}-0\right) \\
\xi_{0}\left(v\left(\xi_{0}+0\right)-v\left(\xi_{0}-0\right)\right) & =(u v)\left(\xi_{0}+0\right)-(u v)\left(\xi_{0}-0\right) .
\end{aligned}
$$

2. Existence of solutions of (1.5), (1.6). To obtain the existence of smooth solutions of $(1.5),(1.6)$, we start with the following altered system:

$$
\begin{gathered}
\varepsilon \ddot{u}=-\xi \dot{u}+2 \mu u \dot{u}, \\
\varepsilon \ddot{v}=-\xi \dot{v}+\mu(\dot{u} v+u \dot{v}), \\
(u(-L), v(-L))=\left(\mu u^{-}, \mu v^{-}\right), \quad(u(L), v(L))=\left(\mu u^{+}, \mu v^{+}\right),
\end{gathered}
$$

with $\mu \in[0,1]$ and $L \geq 1$. The following theorem is a special case of Theorem 3.1 in [1]. 
Theorem 2.1. Assume that there is a constant $M$ depending only on $u^{+}, u^{-}, v^{+}, v^{-}, \varepsilon$ (and thus independent of $\mu$ and $L$ ) such that any solution of (2.1), (2.2) satisfies

$$
\max _{\xi \in[-L, L]}(|u(\xi)|+|v(\xi)|)<M .
$$

Then a solution of (1.5), (1.6) exists.

We remark in passing here that $(2.1),(2.2)$ always has a solution for some $\mu \in(0,1]$ since the mapping $T$ (see the definition in [1]) is compact. Moreover, if the estimate (2.3) holds then (2.1), (2.2) has a solution for all $\mu \in[0,1]$.

Our next goal is to establish the estimate (2.3). To do this we have

Lemma 2.2. Let $(u(\xi), v(\xi))$ be a solution of $(2.1),(2.2)$ on $[-L, L]$ for some $\mu>0$. If $u^{+} \geq u^{-}$, then one of the following holds.

(i) No critical points: $u(\xi), v(\xi)$ have no local maxima or minima on $[-L, L] . u(\xi)$ is constant or strictly increasing while $v(\xi)$ is constant or strictly monotone.

(ii) One critical point: $u(\xi)$ is strictly increasing, and

(a) $v(\xi)$ has a minimum at some $\tau$ with $v(\tau)>0$, or

(b) $v(\xi)$ has a maximum at some $\sigma$ with $v(\sigma)<0$.

Proof. Let us assume that $(u(\xi), v(\xi))$ is a nonconstant solution of $(2.1)$ on $[-L, L]$. We first note that $u(\xi)$ has no critical points in $[-L, L]$. Indeed, suppose $\xi_{0}$ is a critical point of $u(\xi)$. Then $\dot{u}\left(\xi_{0}\right)=0$ and by $(2.1)_{1}$ we have that $u^{(n)}\left(\xi_{0}\right)=0, n \geq 2$ which means $u(\xi)$ is constant in $[-L, L]$. This is a contradiction since $u(\xi)$ is nonconstant.

Now assume that $v(\xi)$ has a minimum at $\tau$. By $(2.1)_{2}$ we get $\dot{u}(\tau) v(\tau)>0$ and so $v(\tau)>0$ since $\dot{u}(\xi)>0$ in $(-L, L)$. We note here that $v(\tau) \neq 0$; otherwise, $\ddot{v}(\tau)=0$. But this is not feasible by uniqueness of solutions of the initial-value problem for (2.1) (see Lemma 4.1 in [1]). Similarly, $v(\sigma)<0$ for a maximum point $\sigma$ of $v(\xi)$. Finally, it is easily seen that $v(\xi)$ has at most one critical point in $[-L, L]$ since $v(\xi)>0$ at a minimum point and $v(\xi)<0$ at a maximum point. The proof is complete.

Lemma 2.3. Let $(u(\xi), v(\xi))$ be a solution of $(2.1),(2.2)$ on $[-L, L]$ for some $\mu>0$. If $u^{+}<u^{-}$, then $u(\xi)$ is strictly decreasing in $[-L, L]$ and $v(\xi)$ satisfies one of the following.

(i) No critical points: $v(\xi)$ is a monotone function on $[-L, L]$;

(ii) One critical point: (a) $v(\xi)$ has a minimum at some $\tau$ with $v(\tau)<0$; (b) $v(\xi)$ has a maximum at some $\sigma$ with $v(\sigma)>0$;

(iii) Two critical points: (a) $v(\xi)$ has a minimum at $\tau$ and a maximum at $\sigma$ with $\tau<\sigma$, and $v(\tau)<0, v(\sigma)>0$; (b) $v(\xi)$ has a maximum at $\sigma$ and a minimum at $\tau$ with $\sigma<\tau$, and $v(\sigma)>0, v(\tau)<0$.

Proof. We only show that $v(\xi)$ has at most two critical points in $[-L, L]$. Indeed, suppose that $v(\xi)$ has three critical points $\sigma_{1}, \tau, \sigma_{2}$ with $\sigma_{1}<\tau<\sigma_{2}$, where $\sigma_{1}, \sigma_{2}$ are the maximum points and $\tau$ is the minimum point of $v(\xi)$ in $[-L, L]$. Then $v\left(\sigma_{1}\right)>0$, $v(\tau)<0$, and $v\left(\sigma_{2}\right)>0$. Thus, there exist two points $\xi_{0} \in\left(\sigma_{1}, \tau\right)$ and $\xi_{1} \in\left(\tau, \sigma_{2}\right)$ such that $v\left(\xi_{0}\right)=v\left(\xi_{1}\right)=0$. Observing that $\dot{v}\left(\xi_{1}\right)>0, \dot{v}\left(\xi_{0}\right)<0$ and $v(\xi)<0$ for $\xi \in\left(\xi_{0}, \xi_{1}\right)$, integrating $(2.1)_{2}$ over $\left(\xi_{0}, \xi_{1}\right)$ we obtain

$$
0<\varepsilon \dot{v}\left(\xi_{1}\right)-\varepsilon \dot{v}\left(\xi_{0}\right)=-\int_{\xi_{0}}^{\xi_{1}} \xi \dot{v}(\xi) d \xi=\int_{\xi_{0}}^{\xi_{1}} v(\xi) d \xi<0
$$


which is a contradiction. The proof is complete.

Now we are in a position to derive the a priori estimate (2.3) required to apply Theorem 2.1. If $u^{+} \geq u^{-},(2.3)$ is trivial by Lemma 2.2. In the following context we assume that $u^{+}<u^{-}$. In this case we know from Lemma 2.3 that $u(\xi)$ is strictly decreasing in $[-L, L]$ and so $u(\xi)$ is uniformly bounded with respect to $\mu \in[0,1], L \geq 1$ and $\varepsilon>0$. It remains to estimate $v(\xi)$ for Cases (ii) and (iii) in Lemma 2.3. (Obviously, $v(\xi)$ is uniformly bounded on $[-L, L]$ with respect to $\mu, L$ for Case (i) in Lemma 2.3.)

LEMma 2.4. For Cases (ii) and (iii) in Lemma $2.3, v(\xi)$ is uniformly bounded on $[-L, L]$ with respect to $\mu \in[0,1]$ and $L \geq 1$.

Proof. Motivated by [2], we first prove the following:

$$
\int_{\alpha}^{\beta} v(\xi) d \xi \geq-(\beta-\alpha) \bar{v}-N
$$

for every interval $(\alpha, \beta) \subset(-L, L)$, where $\bar{v}=\max \left\{\left|v^{+}\right|,\left|v^{-}\right|\right\}$and $N=\bar{v}\left(u^{-}-u^{+}\right)$.

Indeed, we set $\theta_{1}=\sup \{\xi \in[-L, \alpha) \mid v(\xi) \geq-\bar{v}\}$ if $v(\alpha)<-\bar{v}$; on the other hand, we set $\theta_{1}=\inf \{\xi \in(\alpha, \beta) \mid v(\xi) \leq-\bar{v}\}$ if $v(\alpha) \leq-\bar{v}$ (if this set is empty, (2.4) is automatically satisfied). Similarly, we set $\theta_{2}=\inf \{\xi \in(\beta, L] \mid v(\xi) \geq-\bar{v}\}$ if $v(\beta)<-\bar{v}$ while we set $\theta_{2}=\sup \{\xi \in(\alpha, \beta) \mid v(\xi) \leq-\bar{v}\}$ if $v(\beta) \geq-\bar{v}$. Since $v\left(\theta_{1}\right)=v\left(\theta_{2}\right)=-\bar{v}$, we have

$$
\int_{\alpha}^{\beta}(v(\xi)+\bar{v}) d \xi \geq \int_{\theta_{1}}^{\theta_{2}}(v(\xi)+\bar{v}) d \xi=-\int_{\theta_{1}}^{\theta_{2}} \xi \dot{v}(\xi) d \xi
$$

Noting that $\dot{v}\left(\theta_{1}\right) \leq 0, \dot{v}\left(\theta_{2}\right) \leq 0$, we integrate $(2.1)_{2}$ over $\left(\theta_{1}, \theta_{2}\right)$ to obtain

$$
\begin{aligned}
\int_{\alpha}^{\beta}(v(\xi)+\bar{v}) d \xi & \geq \varepsilon \dot{v}\left(\theta_{2}\right)-\varepsilon \dot{v}\left(\theta_{1}\right)+\mu \bar{v}\left(u\left(\theta_{2}\right)-u\left(\theta_{1}\right)\right) \\
& \geq \bar{v}\left(u^{+}-u^{-}\right)=-N
\end{aligned}
$$

which implies (2.4).

Similarly, we have

$$
\int_{\alpha}^{\beta} v(\xi) d \xi \leq(\beta-\alpha) \bar{v}+N
$$

We observe that $v(\xi)$ changes its signs at most one time on any interval $(\alpha, \beta)$. In other words, there is no subinterval $I \subset(\alpha, \beta)$ such that $v(\xi)$ is positive (or negative) immediately to the left and right of $I$ while $v(\xi)$ is negative (or positive) in $I$. Thus, (2.4), (2.5) yield

$$
\int_{\alpha}^{\beta}|v(\xi)| d \xi \leq(\beta-\alpha) \bar{v}+2 N
$$

for every interval $(\alpha, \beta) \subset(-L, L)$.

We now apply $(2.4)$ to estimating $v(\xi)$ from below for Case (ii)(a) in Lemma 2.3. Cases (ii)(b) and (iii) can be treated similarly.

By (2.4) it follows that

$$
-\bar{v}-\frac{N}{|\tau-\xi|} \leq v(\xi) \leq \bar{v}, \quad \xi \in[-L, L] / \tau .
$$


Without loss of generality we assume that $v(\tau)<-\bar{v}$. We fix $\xi_{0}<\tau$ such that $v\left(\xi_{0}\right) \leq-\bar{v}$. For any $\xi \in\left[\xi_{0}, \tau\right)$ we let $\xi^{\prime}$ denote the point in $(\tau, L)$ with the property $v\left(\xi^{\prime}\right)=v(\xi)$ (such a point exists since $v(\xi) \leq-\bar{v}$ ). Integrating $(2.1)$ over $\left(\xi, \xi^{\prime}\right)$ we obtain

$$
\begin{aligned}
\varepsilon \dot{v}\left(\xi^{\prime}\right)-\varepsilon \dot{v}(\xi)= & -\int_{\xi}^{\xi^{\prime}} s \dot{v}(s) d s \\
& +\mu\left(u\left(\xi^{\prime}\right)=u(\xi)\right) v(\xi) .
\end{aligned}
$$

We note that $\dot{v}\left(\xi^{\prime}\right) \geq 0, \dot{v}(\xi) \leq 0$ and $-\int_{\xi}^{\xi^{\prime}} s \dot{v}(s) d s=\int_{\xi}^{\xi^{\prime}}(v(s)-v(\xi)) d s \leq 0$. Therefore, (2.8) gives

$$
\varepsilon \dot{v}(\xi) \geq \mu\left(u(\xi)-u\left(\xi^{\prime}\right)\right) v(\xi) \geq\left(u^{-}-u^{+}\right) v(\xi) .
$$

Integrating the above inequality over $\left(\xi_{0}, \tau\right)$ we deduce that

$$
v(\tau) \geq v\left(\xi_{0}\right) e^{A\left(\tau-\xi_{0}\right)},
$$

where $A=\left(u^{-}-u^{+}\right) / \varepsilon$.

Let $\bar{\xi}<\tau$ such that $v(\bar{\xi})=-\bar{v}$. Then $\xi_{0}$ lies in the interval $[\bar{\xi}, \tau)$. If $\tau-\bar{\xi}<1$, we choose $\xi_{0}=\bar{\xi}$ and by $(2.9), v(\tau) \geq-\bar{v} e^{A}$. On the other hand, if $\tau-\bar{\xi} \geq 1$, we choose $\xi_{0}=\tau-1$. From (2.9), (2.7), it follows that

$$
v(\tau) \geq e^{A\left(\tau-\xi_{0}\right)}\left(-\bar{v}-\frac{N}{|\tau-\xi|}\right)=e^{A}(-\bar{v}-N) .
$$

The proof is complete.

3. Existence of solutions of the Riemann problem: the case when $\left\{v_{\varepsilon}(\xi)\right\}$ is uniformly bounded. In Sec. 2 we have obtained the existence of a smooth solution $\left(u_{\varepsilon}(\xi), v_{\varepsilon}(\xi)\right)$ of $(1.5),(1.6)$ for every $\varepsilon>0$. Moreover, we have the following

LEMMA 3.1. The same results in Lemmas 2.2 and 2.3 are valid for the solution $\left(u_{\varepsilon}(\xi)\right.$; $\left.v_{\varepsilon}(\xi)\right)$ of $(1.5),(1.6)$.

If $\left.\left\{u_{\varepsilon}(\xi), v_{\varepsilon}(\xi)\right) \mid 0<\varepsilon<1\right\}$ is uniformly bounded in $\varepsilon$, we borrow the following result of Dafermos (see Theorem 3.2, [1]) to obtain the existence of a solution to the Riemann problem (1.1), (1.2).

Proposition 3.2. For every fixed $\varepsilon>0$, let $\left(u_{\varepsilon}(\xi), v_{\varepsilon}(\xi)\right)$ be a solution of (1.5), (1.6). Suppose the set $\left\{\left(u_{\varepsilon}(\xi), v_{\varepsilon}(\xi)\right) \mid 0<\varepsilon<1\right\}$ is of uniformly bounded variation. Then $\left\{\left(u_{\varepsilon}(\xi), v_{\varepsilon}(\xi)\right)\right\}$ possesses a subsequence that converges a.e. on $(-\infty, \infty)$ to a function $(u(\xi), v(\xi))$ of bounded variation. The pair $u(x / t), v(x / t)$ provides a weak solution to (1.1), (1.2).

By Lemma 3.1, $u_{\varepsilon}(\xi)$ is always a monotone function on $(-\infty, \infty)$ with the property $\min \left(u^{-}, u^{+}\right) \leq u_{\varepsilon}(\xi) \leq \max \left(u^{-}, u^{+}\right)$for every $\varepsilon>0$, while $\left\{v_{\varepsilon}(\xi) \mid 0<\varepsilon<1\right\}$ is uniformly bounded when $u^{+} \geq u^{-}$or when $v_{\varepsilon}(\xi)$ has no critical points on $(-\infty, \infty)$ for $u^{+}<u^{-}$. So it remains to determine the condition under which $v_{\varepsilon}(\xi)$ is uniformly bounded in $\varepsilon$. In the present situation, $u_{\varepsilon}(\xi)$ is strictly decreasing on $(-\infty, \infty)$ and $v_{\varepsilon}(\xi)$ has one or two critical points on $(-\infty, \infty)$. For definiteness we consider the representative case where $v_{\varepsilon}(\xi)$ is strictly decreasing on $\left(-\infty, \tau_{\varepsilon}\right)$, attains its minimum at $\tau_{\varepsilon}$, and is strictly increasing on $\left(\tau_{\varepsilon}, \infty\right)$. All other cases can be treated similarly.

Let $\tau_{\varepsilon} \rightarrow \tau_{0},\left|\tau_{0}\right| \leq \infty$, as $\varepsilon \rightarrow 0$ (pass to a further subsequence if necessary). 
TheOREM 3.3. If $\left|\tau_{0}\right|=\infty$, then $\left\{v_{\varepsilon}(\xi) \mid 0<\varepsilon<1\right\}$ is uniformly bounded.

Proof. We first assume that $\tau_{0}=\infty$. By (2.7) we get that

$$
-\bar{v}-N \leq v_{\varepsilon}(\xi) \leq \bar{v}
$$

for $\xi \in(-\infty, a]$ and $\varepsilon$ small, where $a$ is any fixed real number. We take $a=2$ and $\varepsilon$ to be so small that $\tau_{\varepsilon}-u_{\varepsilon}\left(\tau_{\varepsilon}\right) \geq 1,\left(\tau_{\varepsilon}-1\right) /\left(\tau_{\varepsilon}-u_{\varepsilon}\left(\tau_{\varepsilon}\right)\right) \leq 2$ (this can be done since $u^{+} \leq u_{\varepsilon}\left(\tau_{\varepsilon}\right) \leq u^{-}$and $\tau_{\varepsilon} \rightarrow \infty$ as $\left.\varepsilon \rightarrow 0\right)$. Integrating $(1.5)_{2}$ over $\left(1, \tau_{\varepsilon}\right)$ and observing $\dot{v}_{\varepsilon}(1) \leq 0$, one finds that

$$
\begin{aligned}
\left(\tau_{\varepsilon}-u_{\varepsilon}\left(\tau_{\varepsilon}\right)\right) v_{\varepsilon}\left(\tau_{\varepsilon}\right) & =\left(1-u_{\varepsilon}(1)\right) v_{\varepsilon}(1)+\int_{1}^{\tau_{\varepsilon}} v_{\varepsilon}(\xi) d \xi-\varepsilon \dot{v}_{\varepsilon}(1) \\
& \geq-(1+\bar{u})(\bar{v}+N)-\left(\tau_{\varepsilon}-1\right) \bar{v}-N
\end{aligned}
$$

by virtue of $(3.1),(2.4)$, where $\bar{u}=\max \left(\left|u^{+}\right|,\left|u^{-}\right|\right)$. Hence, $v_{\varepsilon}\left(\tau_{\varepsilon}\right) \geq-(1+\bar{u})(\bar{v}+N)-$ $N-2 \bar{v}$ for $\varepsilon$ small. Thus, the theorem is proved for $\tau_{0}=\infty$.

Next we suppose that $\tau_{0}=-\infty$. At this time, (3.1) is also valid for $\xi \in[a, \infty), a$ being any number. Thus there exists a point $\xi_{0} \in[1,2]$ such that

$$
0 \leq \dot{v}_{\varepsilon}\left(\xi_{0}\right)=v_{\varepsilon}(2)-v_{\varepsilon}(1) \leq 2 \bar{v}+N
$$

Let $\varepsilon$ be so small that

$$
-\tau_{\varepsilon}+u_{\varepsilon}\left(\tau_{\varepsilon}\right) \geq 1, \quad\left(\xi_{0}-\tau_{\varepsilon}\right) /\left(u_{\varepsilon}\left(\tau_{\varepsilon}\right)-\tau_{\varepsilon}\right) \leq 2
$$

We integrate $(1.5)_{2}$ over $\left(\tau_{\varepsilon}, \xi_{0}\right)$ to obtain that $v_{\varepsilon}\left(\tau_{\varepsilon}\right)$ is uniformly bounded in $\varepsilon$ from below on account of $(3.1)-(3.3),(2.4)$. So the theorem is also true for $\tau_{0}=-\infty$. The proof is complete.

Now we turn to consider the case $\left|\tau_{0}\right|<\infty$. Let $\xi_{\alpha}^{\varepsilon}, \xi_{\beta}^{\varepsilon}$ be the singularity points of (1.5), that is, $\xi_{\alpha}^{\varepsilon}=u_{\varepsilon}\left(\xi_{\alpha}^{\varepsilon}\right)$ and $\xi_{\beta}^{\varepsilon}=2 u_{\varepsilon}\left(\xi_{\beta}^{\varepsilon}\right)$. We set $\xi_{\alpha}=\lim _{\varepsilon \rightarrow 0} \xi_{\alpha}^{\varepsilon}$ and $\xi_{\beta}=\lim _{\varepsilon \rightarrow 0} \xi_{\beta}^{\varepsilon}$. The points $\xi_{\alpha}, \xi_{\beta}$ play an important role in the following discussion.

Lemma 3.4. $\xi_{\beta}$ is defined as above. Then we have $\xi_{\beta}=u^{+}+u^{-}$and for any $\delta>0$,

$$
\lim _{\varepsilon \rightarrow 0} u_{\varepsilon}(\xi)=\left\{\begin{array}{l}
u^{+} \text {uniformly for } \xi>\xi_{\beta}+\delta \\
u^{-} \text {uniformly for } \xi<\xi_{\beta}-\delta
\end{array}\right.
$$

Proof. We take $\xi_{0}=\xi_{\beta}+\delta / 2$. Let $\varepsilon$ be so small that $\xi_{\beta}^{\varepsilon}<\xi_{0}-\delta / 4$. From $(1.5)_{1}$ we have

$$
\dot{u}_{\varepsilon}(\xi)=\dot{u}_{\varepsilon}\left(\xi_{0}\right) \exp \left(\int_{\xi_{0}}^{\xi} \frac{2 u_{\varepsilon}(s)-s}{\varepsilon} d s\right) .
$$

Integrating (3.5) over $\left(\xi_{0}, \xi_{0}+1\right)$, one finds that

$$
u_{\varepsilon}\left(\xi_{0}+1\right)-u_{\varepsilon}\left(\xi_{0}\right)=\dot{u}_{\varepsilon}\left(\xi_{0}\right) \int_{\xi_{0}}^{\xi_{0}+1} \exp \left(\int_{\xi_{0}}^{\xi} \frac{2 u_{\varepsilon}(s)-s}{\varepsilon} d s\right) d \xi
$$


We note that

$$
\begin{aligned}
\int_{\xi_{0}}^{\xi_{0}+1} & \exp \left(\int_{\xi_{0}}^{\xi} \frac{2 u_{\varepsilon}(s)-s}{\varepsilon} d s\right) d \xi \geq \int_{\xi_{0}}^{\xi_{0}+1} \exp \left(\int_{\xi_{0}}^{\xi} \frac{2 u^{+}-s}{\varepsilon} d s\right) d \xi \\
\quad= & \int_{\xi_{0}}^{\xi_{0}+1} \exp \left(\frac{2 u^{+}}{\varepsilon}\left(\xi-\xi_{0}\right)-\frac{1}{2 \varepsilon}\left(\xi^{2}-\xi_{0}^{2}\right)\right) d \xi \\
& \geq \varepsilon \int_{0}^{1} \exp \left(2 u^{+} s-\xi_{0} s-\frac{1}{2} s^{2}\right) d s \triangleq A \cdot \varepsilon
\end{aligned}
$$

Thus, (3.6) gives

$$
0 \geq \dot{u}_{\varepsilon}\left(\xi_{0}\right) \geq \frac{u^{+}-u^{-}}{A \varepsilon}
$$

which combines with (3.5) to yield

$$
\left|\dot{u}_{\varepsilon}(\xi)\right| \leq \frac{u^{-}-u^{+}}{A \varepsilon} \exp \left(\int_{\xi_{0}}^{\xi} \frac{2 u_{\varepsilon}(s)-s}{\varepsilon} d s\right)
$$

Now,

$$
\begin{aligned}
2 u_{\varepsilon}(s)-s & =2\left(u_{\varepsilon}(s)-u_{\varepsilon}\left(\xi_{\beta}^{\varepsilon}\right)\right)+\xi_{\beta}^{\varepsilon}-s \\
& =\left(s-\xi_{\beta}^{\varepsilon}\right)\left(2 \dot{u}_{\varepsilon}\left(\theta_{\varepsilon}\right)-1\right) \leq-\frac{\delta}{4}
\end{aligned}
$$

Therefore,

$$
\left|\dot{u}_{\varepsilon}(\xi)\right| \leq \frac{u^{-}-u^{+}}{A \varepsilon} \exp \left(-\frac{\delta}{4 \varepsilon}\left(\xi-\xi_{0}\right)\right) \text { for } \xi>\xi_{0} .
$$

Let $\xi_{1}>\xi_{\beta}+\delta$. We deduce from

$$
u^{+}-u_{\varepsilon}\left(\xi_{1}\right)=\int_{\xi_{1}}^{\infty} \dot{u}_{\varepsilon}(s) d s
$$

that $\lim _{\varepsilon \rightarrow 0} u_{\varepsilon}\left(\xi_{1}\right)=u^{+}$uniformly by virtue of (3.7). Similarly, one can obtain that $\lim _{\varepsilon \rightarrow 0} u_{\varepsilon}(\xi)=u^{-}$uniformly for $\xi<\xi_{\beta}-\delta$.

Finally, we take $\psi \in C_{0}^{\infty}\left(\xi_{1}, \xi_{2}\right)$, where $\xi_{1}<\xi_{\beta}<\xi_{2}$. By $(1.5)_{1}$ one knows that

$$
\varepsilon \int_{\xi_{1}}^{\xi_{2}} u_{\varepsilon}(\xi) \ddot{\psi}(\xi) d \xi=\int_{\xi_{1}}^{\xi_{2}}\left[u_{\varepsilon}(\xi)(\psi(\xi)+\xi \dot{\psi}(\xi))-u_{\varepsilon}^{2}(\xi) \dot{\psi}(\xi)\right] d \xi
$$

Letting $\varepsilon \rightarrow 0$, we see that

$$
\int_{\xi_{1}}^{\xi_{\beta}}\left[u^{-}(\psi(\xi)+\xi \dot{\psi}(\xi))-\left(u^{-}\right)^{2} \dot{\psi}(\xi)\right] d \xi+\int_{\xi_{\beta}}^{\xi_{2}}\left[u^{+}(\psi(\xi)+\xi \dot{\psi}(\xi))-\left(u^{+}\right)^{2} \dot{\psi}(\xi)\right] d \xi=0
$$

by virtue of (3.4). Therefore,

$$
\left(u^{-}-u^{+}\right)\left(\xi_{\beta}-\left(u^{+}+u^{-}\right)\right) \psi\left(\xi_{\beta}\right)=0
$$

which yields that $\xi_{\beta}=u^{+}+u^{-}$since $u^{+}<u^{-}$and $\psi$ is arbitrary. The proof of the lemma is complete. 
Lemma 3.5. $\xi_{\alpha}$ is defined as above. Then we have

(i) if $u^{-}>u^{+}>0$, then $\xi_{\alpha}=u^{-}, \xi_{\alpha}<\xi_{\beta}$;

(ii) if $u^{+}<u^{-}<0$, then $\xi_{\alpha}=u^{+}, \xi_{\alpha}>\xi_{\beta}$;

(iii) if $u^{-} \geq 0 \geq u^{+}\left(u^{-}>u^{+}\right)$, then $\xi_{\alpha}=\xi_{\beta}=u^{+}+u^{-}$.

Proof. We first show (i) holds. Indeed, $\xi_{\alpha} \leq u^{-}$since $u_{\varepsilon}(\xi) \leq u^{-}$on $(-\infty, \infty)$. Suppose that $\xi_{\alpha}<u^{-}$. Then $\xi_{\alpha}<u^{+}+u^{-}=\xi_{\beta}$. By (3.4) we have that $\lim _{\varepsilon \rightarrow 0} u_{\varepsilon}\left(\xi_{\alpha}^{\varepsilon}\right)=$ $u^{-}$, which is a contradiction. Case (ii) can be treated similarly.

Finally, suppose that $u^{+}<0<u^{-}$. If $\xi_{\alpha}<u^{+}+u^{-}=\xi_{\beta}$, then $\xi_{\alpha}=\lim _{\varepsilon \rightarrow 0} u_{\varepsilon}\left(\xi_{\alpha}^{\varepsilon}\right)=$ $u^{-}$by (3.4), which contradicts $u^{+}<0$. In a similar way $\xi_{\alpha}$ cannot be greater than $u^{+}+u^{-}$. So $\xi_{\alpha}=u^{+}+u^{-}$. The case $u^{+}=0<u^{-}$or $u^{+}<0=u^{-}$is treated similarly and leads to the same result. The proof is complete.

We remark in passing here that some of the results in Lemmas 3.4 and 3.5 were also obtained in [11]. Now we state

Theorem 3.6. $\xi_{\alpha}$ is given in Lemma 3.5. If $\tau_{0} \neq \xi_{\alpha}$, then $\left\{v_{\varepsilon}(\xi) \mid 0<\varepsilon<1\right\}$ is uniformly bounded.

Proof. We only consider Case (i) in Lemma 3.5. Cases (ii) and (iii) are treated similarly. At this time $\xi_{\alpha}=u^{-}$. We distinguish the following cases.

$1^{\circ} . \tau_{0}>\xi_{\alpha}=u^{-}$. We integrate $(1.5)_{2}$ over $\left(\tau_{\varepsilon}, \tau_{0}+1\right)$ to get that

$$
\varepsilon \dot{v}_{\varepsilon}\left(\tau_{0}+1\right)=\left(\tau_{\varepsilon}-u_{\varepsilon}\left(\tau_{\varepsilon}\right)\right) v_{\varepsilon}\left(\tau_{\varepsilon}\right)-\left(\tau_{0}+1-u_{\varepsilon}\left(\tau_{0}+1\right)\right) v_{\varepsilon}\left(\tau_{0}+1\right)+\int_{\tau_{\varepsilon}}^{\tau_{0}+1} v_{\varepsilon}(\xi) d \xi
$$

Observe that $\dot{v}_{\varepsilon}\left(\tau_{0}+1\right) \geq 0$ and $\tau_{\varepsilon}-u_{\varepsilon}\left(\tau_{\varepsilon}\right) \geq \tau_{\varepsilon}-u^{-} \geq \frac{1}{2}\left(\tau_{0}-u^{-}\right)$for $\varepsilon$ small. Therefore,

$$
\begin{aligned}
v_{\varepsilon}\left(\tau_{\varepsilon}\right) & \geq \frac{1}{\tau_{\varepsilon}-u_{\varepsilon}\left(\tau_{\varepsilon}\right)}\left[\left(\tau_{0}+1-u_{\varepsilon}\left(\tau_{0}+1\right)\right) v_{\varepsilon}\left(\tau_{0}+1\right)-\int_{\tau_{\varepsilon}}^{\tau_{0}+1} v_{\varepsilon}(\xi) d \xi\right] \\
& \geq-\frac{2}{\tau_{0}-u^{-}}\left[\left(\tau_{0}+1+\bar{u}\right)(\bar{v}+2 N)+2 \bar{v}+N\right]
\end{aligned}
$$

since $\left|v_{\varepsilon}\left(\tau_{0}+1\right)\right| \leq \bar{v}+2 N$ by $(2.7)$ and $\int_{\tau_{\varepsilon}}^{\tau_{0}+1} v_{\varepsilon}(\xi) d \xi \leq\left(\tau_{0}+1-\tau_{\varepsilon}\right) \bar{v}+N \leq 2 \bar{v}+N$ by (2.5) for $\varepsilon$ small, where $\bar{u}=\max \left\{\left|u^{+}\right|,\left|u^{-}\right|\right\}$. Thus $v_{\varepsilon}\left(\tau_{\varepsilon}\right)$ is uniformly bounded in $\varepsilon$ from below.

$2^{\circ} . \tau_{0}<\xi_{\alpha}=u^{-}$. We integrate $(1.5)_{2}$ over $\left(-\tau_{0}-1, \tau_{\varepsilon}\right)$ to have

$$
\begin{aligned}
-\varepsilon \dot{v}_{\varepsilon}\left(-\tau_{0}-1\right)=\left(-\tau_{\varepsilon}+u_{\varepsilon}\left(\tau_{\varepsilon}\right)\right) & v_{\varepsilon}\left(\tau_{\varepsilon}\right) \\
& -\left(\tau_{0}+1+u_{\varepsilon}\left(-\tau_{0}-1\right)\right) v_{\varepsilon}\left(-\tau_{0}-1\right)+\int_{-\tau_{0}-1}^{\tau_{\varepsilon}} v_{\varepsilon}(\xi) d \xi .
\end{aligned}
$$

We note that $\dot{v}_{\varepsilon}\left(-\tau_{0}-1\right) \leq 0$ and $-\tau_{\varepsilon}+u_{\varepsilon}\left(\tau_{\varepsilon}\right)=-\tau_{\varepsilon}+\xi_{\alpha}^{\varepsilon}-\left(u_{\varepsilon}\left(\xi_{\alpha}^{\varepsilon}\right)-u_{\varepsilon}\left(\tau_{\varepsilon}\right)\right)=$ $\left(\xi_{\alpha}^{\varepsilon}-\tau_{\varepsilon}\right)\left(1-\dot{u}_{\varepsilon}\left(\theta_{\varepsilon}\right)\right) \geq \frac{1}{2}\left(u^{-}-\tau_{0}\right)$ for $\varepsilon$ small. We do as above to have that $v_{\varepsilon}\left(\tau_{\varepsilon}\right)$ is also bounded uniformly in $\varepsilon$. The proof of the theorem is complete.

4. Existence of solutions to the Riemann problem: the case when $\left\{v_{\varepsilon}(\xi)\right\}$ may tend to infinity as $\tau_{0}=\xi_{\alpha}$. In this section we discuss the case when $\left\{v_{\varepsilon}(\xi)\right\}$ may tend to infinity as $\tau_{\varepsilon} \rightarrow \xi_{\alpha}$. We prove that the family $\left\{\left(u_{\varepsilon}(\xi), v_{\varepsilon}(\xi)\right) \mid 0<\varepsilon<1\right\}$ of (1.5), (1.6) can also generate solutions of the Riemann problem (1.1), (1.2). In particular, when $u^{-} \geq 0 \geq u^{+}\left(u^{-}>u^{+}\right)$, Riemann solutions of (1.1), (1.2) contain delta-shock waves. 
Lemma 4.1. Let $\left(u_{\varepsilon}(\xi), v_{\varepsilon}(\xi)\right)$ be a solution of $(1.5),(1.6)$ and let $v_{\varepsilon}(\xi)$ have its minimum at $\tau_{\varepsilon}, \tau_{\varepsilon} \rightarrow \tau_{0}=\xi_{\alpha}$ as $\varepsilon \rightarrow 0$. Then the sequence $\left\{\left(u_{\varepsilon}(\xi), v_{\varepsilon}(\xi)\right) \mid 0<\varepsilon<1\right\}$ possesses a subsequence that converges a.e. on $(-\infty, \infty)$ to functions $(u(\xi), v(\xi))$ satisfying

$$
\begin{gathered}
u^{+} \leq u(\xi) \leq u^{-}, \quad \xi \in(-\infty, \infty), \\
-\bar{v}-\frac{N}{\left|\xi-\tau_{0}\right|} \leq v(\xi) \leq \bar{v}, \quad \xi \in(-\infty, \infty) / \tau_{0}
\end{gathered}
$$

where $\bar{v}=\max \left\{\left|v^{+}\right|,\left|v^{-}\right|\right\}$and $N=\bar{v}\left(u^{-}-u^{+}\right)$.

Proof. Since $u_{\varepsilon}(\xi)$ is strictly decreasing on $(-\infty, \infty),(4.1 \mathrm{a})$ is trivial. By $(2.7), v_{\varepsilon}(\xi)$ is uniformly bounded in $\varepsilon$ on the finite domain $I_{2}=\left[-2, \tau_{0}-\frac{1}{2}\right] \cup\left[\tau_{0}+\frac{1}{2}, 2\right]$ (without loss of generality we assume that $\left|\tau_{0}\right|<1$ ). Applying Helly's theorem there exists a convergent subsequence of $\left\{v_{\varepsilon}(\xi)\right\}$ (still denoted by the original one). Similarly we can extract a convergent subsequence of $\left\{v_{\varepsilon}(\xi)\right\}$ on $I_{3}=\left[-3, \tau_{0}-\frac{1}{3}\right] \cup\left[\tau_{0}+\frac{1}{3}, 3\right]$. Continue this process on each $I_{n}=\left[-n, \tau_{0}-\frac{1}{n}\right] \cup\left[\tau_{0}+\frac{1}{n}, n\right], n=4,5, \ldots$ Finally, extract the diagonal element at each enumerated sequence. The sequence of diagonal elements is convergent at each $\xi \neq \tau_{0}$ to a function $v(\xi)$ defined on $\left(-\infty, \tau_{0}\right) \cup\left(\tau_{0}, \infty\right)$. Also, (4.1b) holds on account of $(2.7)$. Moreover, $v(\xi)$ is locally integrable on $(-\infty, \infty)$ by $(2.6)$ and Fatou's lemma. The proof of the lemma is complete.

The following lemma describes the behavior of $v(\xi)$ at the boundary.

LEMMA 4.2. $v(\xi)$ satisfies the following boundary conditions:

$$
v(-\infty)=v^{-}, \quad v(+\infty)=v^{+} .
$$

Proof. By (2.7),

$$
-\bar{v}-2 N \leq v_{\varepsilon}(\xi) \leq \bar{v} \quad \text { for } \xi \in\left(-\infty,-\xi_{0}\right) \cup\left[\xi_{0}, \infty\right),
$$

where $\xi_{0}=\tau_{0}+1$ and $\varepsilon$ is small.

Let

$$
y_{\varepsilon}(\xi)=\left(\begin{array}{c}
u_{\varepsilon}(\xi) \\
v_{\varepsilon}(\xi)
\end{array}\right), \quad f\left(y_{\varepsilon}\right)=\left(\begin{array}{c}
u_{\varepsilon}^{2}(\xi) \\
u_{\varepsilon}(\xi) v_{\varepsilon}(\xi)
\end{array}\right)
$$

Motivated by [1], we have from (1.5) that

$$
\frac{d}{d \xi}\left(\exp \left(\frac{\xi^{2}}{2 \varepsilon}\right) \dot{y}_{\varepsilon}(\xi)\right)=\frac{1}{\varepsilon} \nabla f\left(y_{\varepsilon}\right) \cdot \dot{y}_{\varepsilon}(\xi) \cdot \exp \left(\frac{\xi^{2}}{2 \varepsilon}\right) .
$$

We integrate $(4.4)$ over $\left(\xi_{0}, \xi\right), \xi>\xi_{0}$, to obtain that

$$
\exp \left(\frac{\xi^{2}}{2 \varepsilon}\right) \dot{y}_{\varepsilon}(\xi)-\exp \left(\frac{\xi_{0}^{2}}{2 \varepsilon}\right) \dot{y}_{\varepsilon}\left(\xi_{0}\right)=\frac{1}{\varepsilon} \int_{\xi_{0}}^{\xi} \nabla f\left(y_{\varepsilon}(s)\right) \dot{y}_{\varepsilon}(s) \exp \left(\frac{s^{2}}{2 \varepsilon}\right) d s .
$$

Using Gronwall's inequality, it follows that

$$
\left|\dot{y}_{\varepsilon}(\xi)\right| \leq\left|\dot{y}_{\varepsilon}\left(\xi_{0}\right)\right| \exp \left(\frac{\xi_{0}^{2}+2 \alpha\left(\xi-\xi_{0}\right)-\xi^{2}}{2 \varepsilon}\right),
$$


where $\alpha=\sup _{\xi \in\left[\xi_{0}, \infty\right)}\left|\nabla f\left(y_{\varepsilon}(\xi)\right)\right|$, which is independent of $\varepsilon$ on account of (4.3).

We note that

$$
\begin{aligned}
\dot{y}_{\varepsilon}\left(\xi_{0}\right) & \int_{\xi_{0}}^{\xi_{0}+1} \exp \left(\frac{\xi_{0}^{2}-\xi^{2}}{2 \varepsilon}\right) d \xi \\
= & y_{\varepsilon}\left(\xi_{0}+1\right)-y_{\varepsilon}\left(\xi_{0}\right)+\frac{1}{\varepsilon} \int_{\xi_{0}}^{\xi_{0}+1} f\left(y_{\varepsilon}(\xi)\right) d \xi \\
& +\frac{1}{\varepsilon} f\left(y_{\varepsilon}\left(\xi_{0}\right)\right) \int_{\xi_{0}}^{\xi_{0}+1} \exp \left(-\frac{\xi^{2}-\xi_{0}^{2}}{2 \varepsilon}\right) d \xi \\
& +\frac{1}{\varepsilon^{2}} \int_{\xi_{0}}^{\xi_{0}+1} \int_{\xi_{0}}^{\xi} s f\left(y_{\varepsilon}(s)\right) \exp \left(\frac{s^{2}-\xi^{2}}{2 \varepsilon}\right) d s d \xi
\end{aligned}
$$

which reduces to

$$
\left|\dot{y}_{\varepsilon}\left(\xi_{0}\right)\right| \leq \text { const } \cdot \varepsilon^{-3}
$$

where the constant is independent of $\varepsilon$. Thus, (4.5) gives

$$
\left|\dot{y}_{\varepsilon}(\xi)\right| \leq \mathrm{const} \cdot \varepsilon^{-3} \exp \left(\frac{\xi_{0}^{2}+2 \alpha\left(\xi-\xi_{0}\right)-\xi^{2}}{2 \varepsilon}\right), \quad \xi>\xi_{0} .
$$

Therefore, for $\xi>\max \left(\xi_{0}, \alpha+\left|\alpha-\xi_{0}\right|\right)(4.6)$ shows that $\left|\dot{v}_{\varepsilon}(\xi)\right| \rightarrow 0$ as $\varepsilon \rightarrow 0$. We recall that $v_{\varepsilon}(\xi)$ converges pointwise to $v(\xi)$ for $\xi>\xi_{0}$. Thus $v(\xi)$ must be a constant for $\xi>\max \left(\xi_{0}, \alpha+\left|\alpha-\xi_{0}\right|\right)$. Since, for any $\varepsilon>0, \lim _{\xi \rightarrow \infty} v_{\varepsilon}(\xi)=v^{+}$, the constant must be $v^{+}$. A similar argument holds for $\xi=-\infty$. The proof is complete.

By Lemma 3.4 we know that only $\xi_{\beta}=\sigma=u^{+}+u^{-}$is a discontinuity point of $u(\xi)$ on $(-\infty, \infty)$, i.e., $u(\xi)=u(x, t)$ has a shock wave with speed $\sigma=u^{+}+u^{-}\left(u^{-}>u^{+}\right)$ in the $(x, t)$-plane. In the following we address that the discontinuity points of $v(\xi)$ on $(-\infty, \infty)$ are just the points $\xi_{\alpha}, \xi_{\beta}$, the limits of singularity points of the ordinary differential equations (1.5).

TheOrem 4.3. Assume that $\tau_{0}=\xi_{\alpha}\left(u^{-}>u^{+}\right)$. Then for each $\delta>0$,

$$
v(\xi)= \begin{cases}v^{-}, & \xi<u^{-}-\delta \\ v_{1}, & u^{-}+\delta<\xi<\sigma-\delta \quad \text { for } u^{-}>u^{+}>0 \\ v^{+}, & \sigma+\delta<\xi\end{cases}
$$

where $v_{1}=u^{-} v^{+} / u^{+}, \sigma=u^{+}+u^{-}$.

$$
v(\xi)= \begin{cases}v^{-}, & \xi<\sigma-\delta \\ v_{2}, & \sigma+\delta<\xi<u^{+}-\delta \quad \text { for } u^{+}<u^{-}<0 \\ v^{+}, & u^{+}+\delta<\xi\end{cases}
$$

where $v_{2}=u^{+} v^{-} / u^{-}$.

$$
v(\xi)=H(\xi-\sigma)+s \cdot \delta(\xi-\sigma) \text { for } u^{+} \leq 0 \leq u^{-},
$$


where

$$
H(x)=\left\{\begin{array}{ll}
v^{-}, & x<0 \\
v^{+}, & x>0
\end{array},\right.
$$

$\delta(x)$ is the Dirac function supported at $x=0$, and $s$ is the strength of $\delta(x)$ with $s=$ $u^{-} v^{+}-u^{+} v^{-}$.

Proof. We first prove Case (i). At this time $\xi_{\alpha}=u^{-}<\xi_{\beta}=\sigma$. We take $\xi_{1}, \xi_{2}$ with the property $-\infty<\xi_{1}<\xi_{2}<u^{-}-\delta$. For each $\psi \in C_{0}^{1}\left(\xi_{1}, \xi_{2}\right)$, we have from $(1.5)_{2}$ that

$$
\varepsilon \int_{\xi_{1}}^{\xi_{2}} v_{\varepsilon}(\xi) \ddot{\psi}(\xi) d \xi=\int_{\xi_{1}}^{\xi_{2}} v_{\varepsilon}(\xi)\left(\xi \dot{\psi}(\xi)+\psi(\xi)-u_{\varepsilon}(\xi) \dot{\psi}(\xi)\right) d \xi
$$

By (2.7)

$$
-\bar{v}-\frac{2 N}{\delta} \leq v_{\varepsilon}(\xi) \leq \bar{v} \quad \text { for } \xi \in\left(\xi_{1}, \xi_{2}\right), \quad \varepsilon \text { small. }
$$

Letting $\varepsilon \rightarrow 0$ in (4.7), using (2.6), (4.8), (3.4), and the Lebesgue dominated convergence theorem, one obtains that

$$
\int_{\xi_{1}}^{\xi_{2}} v(\xi)\left(\xi \dot{\psi}(\xi)+\psi(\xi)-u^{-} \dot{\psi}(\xi)\right) d \xi=0 \quad \text { for each } \psi \in C_{0}^{1}\left(\xi_{1}, \xi_{2}\right)
$$

which gives

$$
\int_{\xi_{1}}^{\xi_{2}} v(\xi) \dot{\phi}(\xi) d \xi=0 \quad \text { for each } \phi(\xi) \in C_{0}^{1}\left(\xi_{1}, \xi_{2}\right)
$$

by setting $\phi(\xi)=\psi(\xi) /\left(\xi-u^{-}\right)$since $\xi-u^{-} \neq 0$ for $\xi \in\left(\xi_{1}, \xi_{2}\right)$. Therefore, $v(\xi)$ is a constant on $\left(-\infty, u^{-}-\delta\right)$ and thus $v(\xi)=v^{-}$since $v(-\infty)=v^{-}$by (4.2). Similarly, we have $v(\xi)=u^{+}$for $\xi>\sigma+\delta$. By the same procedure as above we claim that $v(\xi)$ is also a constant, denoted by $v_{1}$, on $\left(u^{-}+\delta, \sigma-\delta\right)$. In view of the Rankine-Hugoniot condition (1.8): $\left(u^{+}+u^{-}\right)\left(v^{+}-v_{1}\right)=u^{+} v^{+}-u^{-} v_{1}, v_{1}=u^{-} v^{+} / u^{+}$. Case (ii) can be treated similarly. We now turn to prove Case (iii).

Just as we did above, we have that

$$
v(\xi)=v^{-} \quad \text { for } \xi<\sigma-\delta, \quad v(\xi)=v^{+} \text {for } \xi>\sigma+\delta,
$$

and $(u(\xi), v(\xi))$ share the same discontinuity point $\xi=\sigma=u^{+}+u^{-}$. But the RankineHugoniot condition is not satisfied for $v(\xi)$, that is, $\left(u^{+}+u^{-}\right)\left(v^{+}-v^{-}\right) \neq u^{+} v^{+}-u^{-} v^{-}$ if $v^{+} u^{-} \neq u^{+} v^{-}$. Thus we require $v(\xi), u(\xi)$ to take "certain values" at $\xi=\sigma$. To accomplish this, we take $\phi \in C_{0}^{\infty}\left(\xi_{1}, \xi_{2}\right), \xi_{1}<\sigma<\xi_{2}$ and $\phi(\xi)=\phi(\sigma)$ for $\xi$ in a small neighborhood of the point $\sigma$. By (4.7) we get that

$$
\lim _{\varepsilon \rightarrow 0} \int_{\xi_{1}}^{\xi_{2}} v_{\varepsilon}(\xi)\left(\xi \dot{\phi}(\xi)+\phi(\xi)-u_{\varepsilon}(\xi) \dot{\phi}(\xi)\right) d \xi=0
$$


For $\alpha_{1}, \alpha_{2}$ near $\sigma, \alpha_{1}<\sigma<\alpha_{2},(4.9),(3.4)$ reduce to

$$
\begin{aligned}
\lim _{\varepsilon \rightarrow 0} \int_{\xi_{1}}^{\xi_{2}} v_{\varepsilon}(\xi)\left(\xi-u_{\varepsilon}(\xi)\right) \dot{\phi}(\xi) d \xi \\
=\lim _{\varepsilon \rightarrow 0}\left[\int_{\xi_{1}}^{\alpha_{1}} v_{\varepsilon}(\xi)\left(\xi-u_{\varepsilon}(\xi)\right) \dot{\phi}(\xi) d \xi\right. \\
\left.\quad+\int_{\alpha_{2}}^{\xi_{2}} v_{\varepsilon}(\xi)\left(\xi-u_{\varepsilon}(\xi)\right) \dot{\phi}(\xi) d \xi\right] \\
=\int_{\xi_{1}}^{\alpha_{1}} v^{-}\left(\xi-u^{-}\right) \dot{\phi}(\xi) d \xi+\int_{\alpha_{2}}^{\xi_{2}} v^{+}\left(\xi-u^{+}\right) \dot{\phi}(\xi) d \xi \\
=\left(v^{+} u^{+}-v^{-} u^{-}-\left(v^{+} \alpha_{2}-v^{-} \alpha_{1}\right)\right) \phi(\sigma) \\
\quad-\int_{\xi_{1}}^{\alpha_{1}} v^{-} \phi(\xi) d \xi-\int_{\alpha_{2}}^{\xi_{2}} v^{+} \phi(\xi) d \xi .
\end{aligned}
$$

Letting $\alpha_{1} \rightarrow \sigma-, \alpha_{2} \rightarrow \sigma+$, we have that

$$
\begin{aligned}
& \lim _{\varepsilon \rightarrow 0} \int_{\xi_{1}}^{\xi_{2}} v_{\varepsilon}(\xi)\left(\xi-u_{\varepsilon}(\xi)\right) \dot{\phi}(\xi) d \xi=\left(u^{+} v^{-}-u^{-} v^{+}\right) \phi(\sigma) \\
& \quad+\int_{\xi_{1}}^{\xi_{2}} H(\xi-\sigma) \phi(\xi) d \xi
\end{aligned}
$$

where

$$
H(x)= \begin{cases}v^{-}, & x<0 \\ v^{+}, & x>0 .\end{cases}
$$

Therefore, (4.10), (4.11) yield that

$$
\lim _{\varepsilon \rightarrow 0} \int_{\xi_{1}}^{\xi_{2}} v_{\varepsilon}(\xi) \phi(\xi) d \xi=\phi(\sigma)\left(u^{-} v^{+}-u^{+} v^{-}\right)+\int_{\xi_{1}}^{\xi_{2}} H(\xi-\sigma) \phi(\xi) d \xi .
$$

By the approximation process, (4.12) holds for all $\phi \in C_{0}^{\infty}\left(\xi_{1}, \xi_{2}\right)$. Thus, we may define $v(\xi)=H(\xi-\sigma)+s \cdot \delta(\xi-\sigma)$ on $(-\infty, \infty)$. We now determine the value of $u(\xi)$ at $\xi=\sigma$.

Let $\xi_{1}<\sigma<\xi_{2}, \phi \in C_{0}^{\infty}\left(\xi_{1}, \xi_{2}\right)$. By $(1.7)_{2}$ we have that

$$
\int_{\xi_{1}}^{\xi_{2}} v(\xi)(\xi \dot{\phi}(\xi)+\phi(\xi)-u(\xi) \dot{\phi}(\xi)) d \xi=0
$$

that is,

$$
\int_{\xi_{1}}^{\xi_{2}}(H(\xi-\sigma)+s \cdot \delta(\xi-\delta))(\xi \dot{\phi}(\xi)+\phi(\xi)-u(\xi) \dot{\phi}(\xi)) d \xi=0 .
$$

Therefore,

$$
\left(u^{-} v^{+}-u^{+} v^{-}\right)(\sigma-u(\sigma)) \dot{\phi}(\sigma)=0,
$$

which reduces to $u(\sigma)=\sigma=u^{+}+u^{-}$since $u^{-} v^{+}-u^{+} v^{-} \neq 0$ and $\phi$ is arbitrary. The proof of the theorem is complete.

We remark here that some of the results in Theorem 4.3 were obtained by Tan, Zhang, and Zheng [11] by considering the limiting behavior of solutions to (1.3), (1.6). 
Acknowledgment. The author wishes to thank Professor Xiaqi Ding for suggesting that he consider this problem and also Professor Y. G. Lu for helpful discussion. The research of the author was supported in part by the Chinese Young National Science Foundation.

\section{REFERENCES}

[1] C. M. Dafermos, Solution of the Riemann problem for a class of hyperbolic systems of conservation laws by the viscosity method, Arch. Rat. Mech. Anal. 52, 1-9 (1973)

[2] C. M. Dafermos and R. J. DiPerna, The Riemann problem for certain classes of hyperbolic systems of conservation laws, J. Differential Equations 20, 90-114 (1976)

[3] H. T. Fan, A vanishing viscosity approach on the dynamics of phase transitions in van der Waals fluids, J. Differential Equations 103, 179-204 (1993)

[4] D. Gilbarg and N. S. Trudinger, Elliptic Partial Differential Equations of Second Order, 2nd edition, Springer-Verlag, 1983

[5] B. L. Keyfitz and H. C. Kranzer, A viscosity approximation to system of conservation laws with no classical Riemann solutions, Nonlinear Hyperbolic Problem, Lecture Notes in Math., Vol. 1402, Springer-Verlag, NY, 1989, pp. 185-197

[6] D. J. Korchinski, Solution of a Riemann problem for a $2 \times 2$ system of conservation laws possessing no classical weak solution, Ph.D. thesis, Adelphi University, 1977

[7] M. Slemrod, A limiting "viscosity" approach to the Riemann problem for materials exhibiting change of phase, Arch. Rat. Mech. Anal. 41, 327-366 (1989)

[8] M. Slemrod and A. E. Tzavaras, A limiting viscosity approach for the Riemann problem in isentropic gas dynamics, Indiana Univ. Math. J. 4, 1047-1074 (1989)

[9] V. A. Tupciev, On the method of introducing viscosity in the study of problems involving decay of a discontinuity, Dokl. Akad. Nauk SSR 211, 55-58 (1973); translated in Soviet Math. Dokl. 14

[10] D. C. Tan and T. Zhang, Two-dimensional Riemann problem for a hyperbolic system on nonlinear conservation laws, Acta Math. Sci. 11, 369-392 (1991)

[11] D. C. Tan, T. Zhang, and Y. X. Zheng, Delta-shock waves as limits of vanishing viscosity for hyperbolic systems of conservation laws, J. Differential Equations 112, 1-32 (1994) 